\title{
Normal Mode Analysis of The Thermal conductivity in Amorphous Polymers: The Importance of Localized Modes
}

\section{Buxuan Li}

Massachusetts Institute of Technology https://orcid.org/0000-0002-7501-6125

\section{Freddy DeAngelis}

Massachusetts Institute of Technology

Gang Chen

Massachusetts Institute of Technology

Asegun Henry ( $\square$ ase@mit.edu )

Massachusetts Institute of Technology

\section{Article}

Keywords: thermal conductivity, amorphous polymer, localization, normal modes, molecular dynamics, lattice dynamics

Posted Date: October 28th, 2021

DOI: https://doi.org/10.21203/rs.3.rs-966158/v1

License: (c) (i) This work is licensed under a Creative Commons Attribution 4.0 International License. Read Full License 


\title{
Normal Mode Analysis of the Thermal conductivity in Amorphous Polymers: The Importance of Localized Modes
}

\author{
Buxuan $\mathrm{Li}^{1}$, Freddy DeAngelis ${ }^{2}$, Gang Chen ${ }^{1}$, Asegun Henry ${ }^{1,2}$ \\ ${ }^{1}$ Department of Mechanical Engineering, Massachusetts Institute of Technology, \\ Cambridge MA, 02139, USA \\ ${ }^{2}$ Woodruff School of Mechanical Engineering, Georgia Institute of Technology, \\ Atlanta, GA 30332, USA
}

\begin{abstract}
Polymers are a unique class of materials from the perspective of normal mode analysis. Polymers consist of individual chains with repeating units and strong intra-chain covalent bonds, and amorphous arrangements among chains with weak inter-chain van der Waals and for some polymers also electrostatic interactions. Intuitively, this strong heterogeneity in bond strength can give rise to interesting features in the constituent phonons, but such effects have not been studied deeply before. Here, we use lattice dynamics and molecular dynamics to perform modal analysis of the thermal conductivity in amorphous polymers for the first time. We find an abnormally large population of localized modes in amorphous polymers, which is dramatically different from amorphous inorganic materials. Contrary to the common picture of thermal transport, localized modes in amorphous polymers are found to be the dominant contributors to thermal conductivity. We find that a significant portion of the localization happens within individual chains, but heat is dominantly conducted when localized modes involve two chains. These results suggest that even though each polymer is different, localized modes play a key role. The results provide new perspective on why polymer thermal conductivity is generally quite low and gives insight into how to potentially change it.
\end{abstract}

Keywords: thermal conductivity, amorphous polymer, localization, normal modes, molecular dynamics, lattice dynamics

\section{Introduction}

Polymers are one of the most important and widely used classes of materials in modern society. The proliferation of polymers is attributed to their low density, low cost, high resistance to corrosion and the low-energy requirements to manufacture/form them into requisite shapes. In their usage, polymers are commonly amorphous in structure and have thermal conductivities on the order of $0.1 \mathrm{~W} \mathrm{~m}^{-1} \mathrm{~K}^{-1}$. The low thermal conductivity (TC) of polymers is useful in many contexts (e.g., food containers and structural materials), but also becomes a limitation in other contexts (e.g., electronics packaging, heat exchangers etc.). Prior work ${ }^{1-11}$ showing that the TC of polymers can be increased by two orders of magnitude to the same range as most metals, has prompted interest in understanding and engineering the TC of polymers for new applications, such as heat exchangers $^{12}$ and thermally conductive chassis for personal electronics. ${ }^{13,14}$ 
While the low value of TC for amorphous polymers is generally understood to be associated with their disordered structure, amorphous polymer TC is still an order of magnitude lower than that of amorphous inorganic materials, and the reason why is not entirely clear from a theoretical perspective. For crystalline materials, the TC is usually well explained by the phonon gas modal (PGM). ${ }^{15-17}$ However, there is growing evidence to suggest that the PGM is insufficient for understanding the thermal behavior of disordered materials ${ }^{18-39}$, such as an amorphous polymer. The structural or compositional disorder within disordered materials causes a drastic change in the character of vibrational modes, which are no longer spatially periodic, as they are in crystalline materials. ${ }^{40}$ As a result, the PGM can become ill-suited for describing the behaviors and contributions of phonons/normal modes with non-periodic mode shapes, which we will henceforth refer to as "non-propagating".

When a significant fraction of the modes in a material cease to have propagating character, the PGM can fail at describing the TC in ways that cannot be corrected with simple modifications of terms that treat it as a perturbation. ${ }^{41}$ This is because there is an intrinsic inapplicability of the PGM physical picture to modes that are non-propagating, since a well-reasoned group velocity or wave vector cannot be assigned to nonpropagating modes. ${ }^{21}$ For amorphous materials, Allen-Feldman (AF) proposed a model based on supercell lattice dynamics calculations that naturally classifies modes into three distinct categories, namely propagons, diffusons, and locons. ${ }^{19,42,43}$

Propagons are modes that most closely resemble the traditional picture of a phonon, which arises from the periodicity in crystalline materials. Propagons have a well-defined wave vector and a periodic mode shape that can produce a propagating wave packet, ${ }^{44}$ and we therefore refer to them herein as propagating. They tend to be low-frequency modes that are delocalized throughout the entire system, and their propagating nature is what allows them to be accurately described by the PGM. Diffusons, while spatially delocalized, exhibit no apparent periodicity in the atomic vibrations/mode shape. Instead, these vibrations appear random, and it is not clear how one could define a wave-vector and/or a group velocity for them. Thus, diffusons are not likely to be well described by the PGM. Finally, locons are spatially localized modes that exist in a portion of the supercell that have their energy highly concentrated onto a subset of atoms. In all previous studies, locons have comprised the smallest group of modes $21,35,41,45$ and they have been assumed to be too localized to contribute to TC in a significant way, ${ }^{19,21,30,46-50}$ although there has been recent evidence that this may not always be the case. ${ }^{35}$

The AF framework, which describes the contributions of diffusons, has been successfully applied to the explain TC of glasses, ${ }^{49-61}$ amorphous semiconductors, ${ }^{24,44,45,62-69}$ solid solutions, ${ }^{70,71}$ organic compounds ${ }^{72-74}$ composites, ${ }^{75,76}$ phase change materials, ${ }^{77}$ and low-dimension materials. ${ }^{78,79}$ However, the AF method neglects the contributions of locons altogether. The AF method was extended by Lv and Henry, and termed the Green Kubo Mode Analysis (GKMA) $21,22,35,80-82$ method. GKMA combines the normal modes obtained from supercell lattice dynamics (LD), obtained in the harmonic limit, with the anharmonic trajectory obtained from a molecular dynamics (MD) simulation. This allows

46 one to quantify the contributions from all three classes of modes (i.e., propagons, 
diffusons and locons), all in a single and consistent framework with anharmonicity. As a result, GKMA provides a more complete description of mode level TC and can provide useful comparisons to experiments when a quantum correction is used. $35,80,81,83$

Spatially extended modes can be distinguished from spatially localized modes using the participation ratio (PR), and the extended modes i.e., propagons and diffusons, can be distinguished from each other, using a method developed by Seyf and Henry. ${ }^{44}$ It should be noted that a variety of other approaches also exist to distinguish between propagons and diffusons. Yet, despite significant progress on developing modal analysis methods, there is little, if any, literature reporting their application to resolve the modal contributions to $\mathrm{TC}$ in amorphous polymers.

Polymers are special because they can have both strong intra-chain covalent bonds and weak interchain Van der Waals bonds, and for some polymers, electrostatic interactions across chains. From the perspective of normal modes, they're a unique class of materials, because individual chains have repeating structures even the chains are randomly arranged. Besides, via mechanically stretching, it is possible to align the polymer chains without chemical modification to achieve a more ordered structure. ${ }^{73,76,77}$ Thus, polymers provide a means for studying how disorder affects phonon transport in a manner whereby one could theoretically observe a continuous transition from disordered to ordered - all in a single sample. To better understand the physics of phonons/normal modes in polymers, herein we used GKMA, and to the best of our knowledge, this is the first-time supercell LD has been used to study the normal modes in amorphous polymers.

\section{Modeling and Simulations}

We performed LD calculations on well-relaxed amorphous polymers to determine the vibrational normal modes. Mode resolved TC was calculated using GKMA with equilibrium MD simulations and the details associated with the relaxation procedure, interatomic potential, and LD/MD are provided in the supplementary information. Three amorphous thermoplastics are studied: amorphous poly(methyl methacrylate) (a-PMMA), amorphous polystyrene (a-PS), and amorphous polyvinyl chloride (a-PVC). All three polymers contain carbon $(\mathrm{C})$ and hydrogen $(\mathrm{H})$, while PMMA contains oxygen $(\mathrm{O})$ atoms, and PVC contains chlorine $(\mathrm{Cl})$. The molecular structure of each monomer is shown below in Fig. 1. 

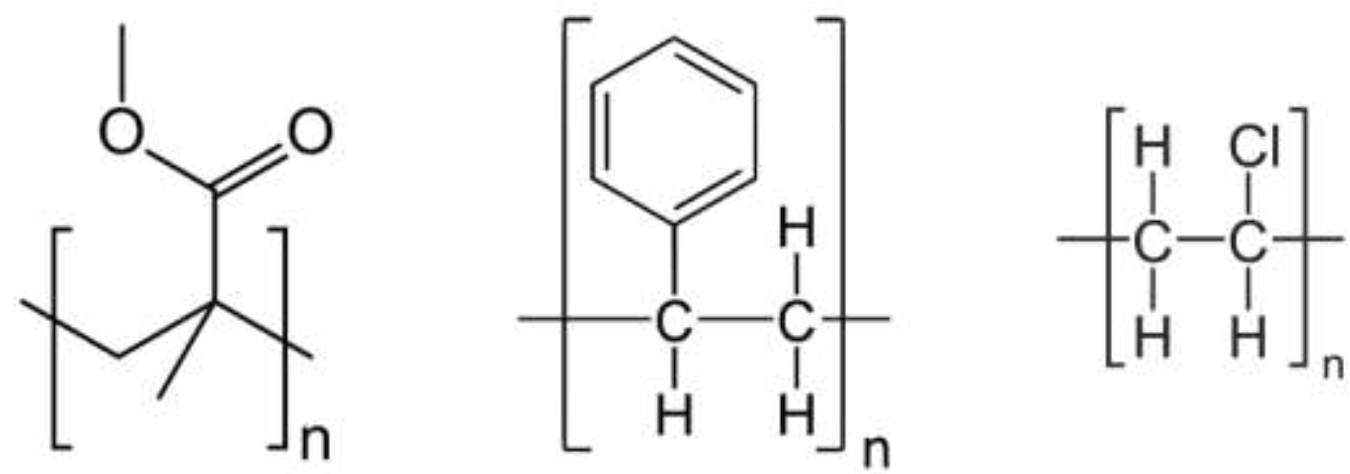

Figure 1| Chemical structures of thermoplastics in study: a-PMMA (left), a-PS (center), and a-PVC (right).

We chose to study these three thermoplastics for two primary reasons: 1) Due to the nature of thermoplastics, they can be annealed and/or melted using MD to relax the structure without concern about significant changes to the chemical bonding, as opposed to thermosets, which are irreversibly cured. 2) Since there is a practical limit to the number of polymers that can be studied herein, given finite computational resources, we attempted to study thermoplastics with some significant heterogeneity in monomer structure/chemistry. By focusing on these thermoplastics, there will perhaps be some observations that may apply more broadly to thermoplastics in general. Due to the significant differences between the three thermoplastics selected, the findings that hold across all three polymers may have implications for a large number of other thermoplastics, as opposed to the alternative of studying three very chemically similar thermoplastics.

To characterize the normal modes in amorphous polymers, we studied the normal modes cumulative density of states (DOS) with different measures of localization in Fig 2. Figure $2 a$ shows the PR versus mode frequency. The PR measures how many atoms are actively involved in a given mode, and therefore is a measure of the degree of localization. The definition of PR can be found in supporting information. Surprisingly, as compared to the modes in amorphous silicon, ${ }^{19,44,80}$ which are similar to the modes in amorphous carbon $^{81}$ and amorphous germanium, the modes in amorphous polymers are much more localized than any of the aforementioned amorphous semiconductors. The modes in the polymers studied here have extremely low PR across a broad range of frequencies as shown in Fig. 2a. Based on the PR, the vast majority of the modes are considered locons using the criterion $\mathrm{PR}<0.1$, which is more conservative than the widely used delineation criterion of 0.2 in other studies, ${ }^{41,43,44,84}$ to classify modes as locons. Interestingly, if one were to adopt this less conservative criterion, almost every mode could be considered a locon with PR $<0.2$ (i.e., $82 \%, 95 \%$, and $98 \%$ of the modes in a-PMMA, a-PS, and a$\mathrm{PVC}$, respectively). 

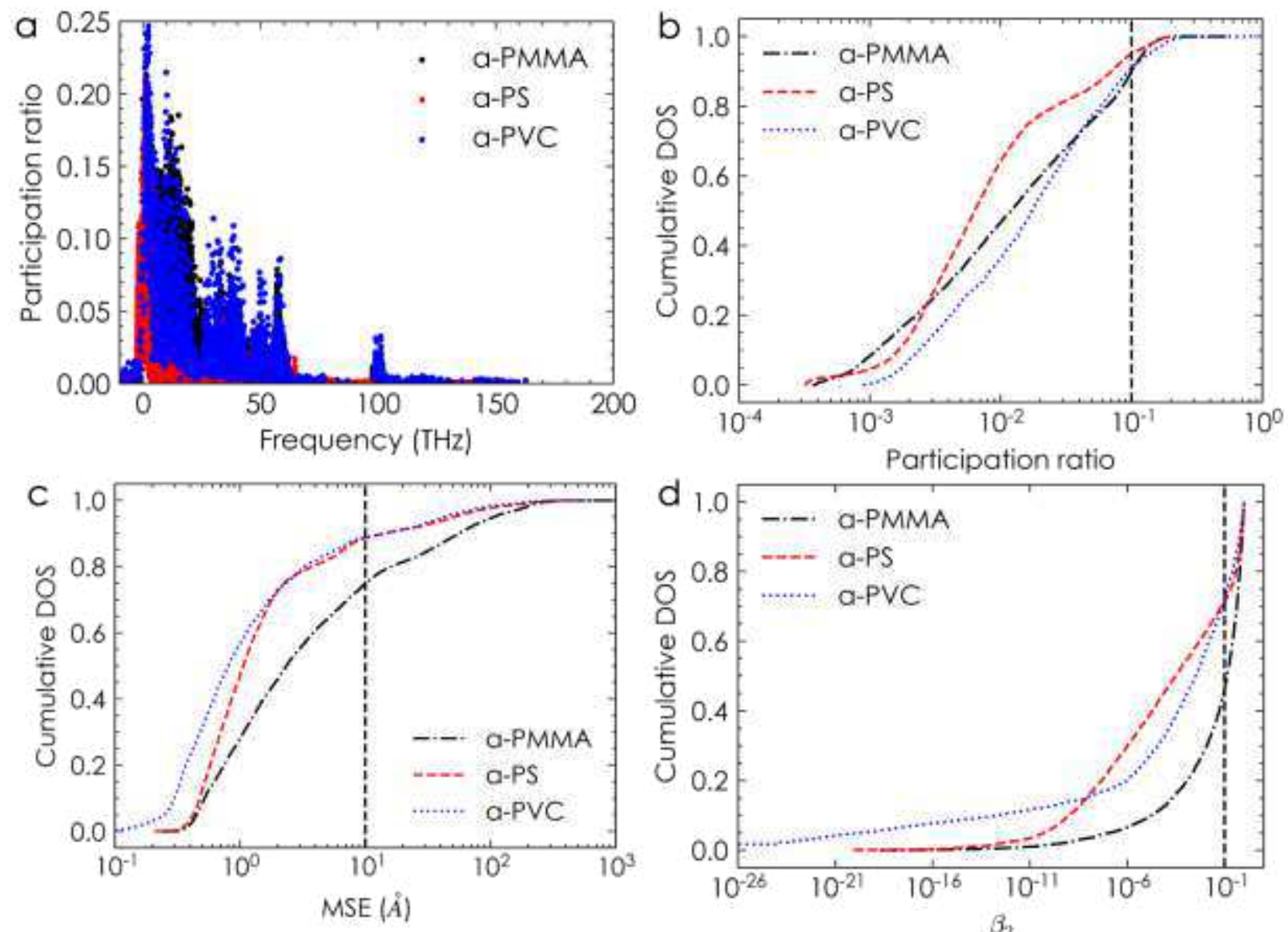

Figure 2| Normal modes in amorphous polymers from LD. The results shown come from supercells of a-PMMA with 3000 atoms, a-PS with 3000 atoms, and a-PVC with 1000 atoms. Panel a shows the PR versus frequency for all three polymers. Panel b, $c$, and $d$ show the cumulative DOS with respect to PR, MSE, and $\beta_{2}$, respectively. The vertical lines in panel $b$ and $c$ show the delineation between highly localized modes and others, while the vertical line in panel d shows the delineation between modes confined to a single chain vs multiple chains. (Colors online)

Such a high degree of localization, especially at low frequencies has not been observed in any other bulk material to date. However, when visualizing the eigenvectors, which describe the square root mass weighted displacement of each atom, as it participates in a given mode, many of the locons seem to have their eigen vectors spread over a significant portion of the supercell. Several examples of modes with low PR yet high spatial extent for a-PMMA are shown in Supplementary Information (SI) Fig. S2. To better quantify the characteristics of these modes, we created a new descriptor that measures the spatial extension of a mode, termed the mode spatial extent (MSE). MSE measures the size of the spatial region over which participating atoms are distributed, using a unit of length. MSE takes both the eigenvectors and the corresponding atom coordinates into consideration, while PR only measures what fraction of the atoms contain the mode energy. Therefore, MSE describes whether the excited atoms of one mode are spreading over the entire chain/simulation domain or spatially confined to a small region e.g. a 
functional group. For each mode, the atomic vibrational amplitude profile with respect to spatial coordinate $x($ and $y, z)$ is fitted as a two-term Gaussian $\left(f(x)=a_{1} e^{-\left(\frac{x-b_{1}}{c_{1}}\right)^{2}}+\right.$ $\left.a_{2} e^{-\left(\frac{x-b_{2}}{c_{2}}\right)^{2}}\right)$, where $f(x)$ is the vibrational amplitude at position $x$, and $a, b$ are constants. The width of the mode in $x$ direction is calculated as as $\left(c_{1}+c_{2}\right) / 2$. The MSE of one mode is the average of the fitted width in three directions. A Gaussian was chosen based on inspection of the modes, but different trial functions other than a two-term Gaussian were also used and the resulting MSE was not sensitive to the choice of trial function. MSE in essence attempts to quantify how large a mode is, by measuring the size of the region to the participating atoms occupy. Note that the calculation of MSE involves fitting a twoterm Gaussian, which might yield values greater than the simulation domain. This is reasonable as in the ideal case the MSE of a periodic mode is infinite. The details of MSE calculation can be found in SI.

In the ensuing analysis, MSE is used as a complimentary descriptor to PR for characterization of mode localization. It should be noted that there are no strict thresholds for either PR or MSE in mode characterization. For PR, the value 0.2 have been suggested for identifying locons, ${ }^{44,45,79,84}$ and 0.1 is used in this study as a more conservative PR cut-off in identification of locons. For MSE, in cases where the MSE is on the order of the atomic spacing or lower $(\sim 1 \AA)$, the mode can be considered highly spatially localized, while a MSE of $10 \AA$ is closer to the order of the dimensions of the supercell in this work. In Fig. S2, several modes are shown that have low PR ( 0.01) yet large MSE $(\sim 100 \AA)$ in a-PMMA. Despite the way the eigenvectors appear upon inspection, the cumulative DOS vs. MSE in Fig. $2 \mathrm{c}$ confirms that fact that the majority of modes are in fact spatially localized, with a MSE $<10 \AA$. For a-PMMA, a-PS, and a-PVC, the factions of modes with MSE $<10 \AA$ are $75 \%, 89 \%$, and $89 \%$, respectively.

In Fig. 3, we show the MSE and PR for all three polymers. While generally modes with smaller PR will have a smaller MSE, these results also show that modes with extremely low PR can still have a relatively large MSE, which visually corresponds to some degree of spreading as shown in the examples in Fig. S2. If we combine these two measures, it suggests that there could be a significant amount of locons in amorphous polymers involving atoms over an extended spatial region. In the case of locons $(P R<0.1)$ in aPMMA, the MSE calculations indicate $25 \%$ of these modes are spatially spread over more than $10 \AA$ (MSE > $10 \AA$ ) and $47 \%$ of these modes are partially spread with MSE values between $1-10 \AA$. Importantly, the results for a-PS and a-PVC are similar, which suggests this may be a general feature of amorphous polymers. For locons in a-PS, $11 \%$ have an MSE greater than $10 \AA$, and $41 \%$ have an MSE between 1 and $10 \AA$. For locons in aPVC, $14 \%$ have an MSE greater than $10 \AA$, and $58 \%$ have an MSE between 1 and $10 \AA$.

Interestingly, both descriptors offer insights. If a few atoms have eigenvectors much larger than the remainder of the atoms in the supercell, it leads to a low PR. However, if those atoms are separated by a significant distance and/or the remaining atoms exhibit small participation in the mode over a larger region, it can result in a larger MSE. It seems reasonable to postulate that similar results might also be observed for other thermoplastics, and maybe even thermosets. Nonetheless, more materials need to be 
studied to confirm or deny this hypothesis. Regardless, the difference between the two descriptors is an indication that perhaps "locons" (specifically, modes with PR $<0.1$ and high MSE > $10 \AA$ ) may be able to interact with other modes more than previously thought, thereby - contrary to the findings of many previous studies, ${ }^{47-50}$ playing an important role 5 in thermal transport.
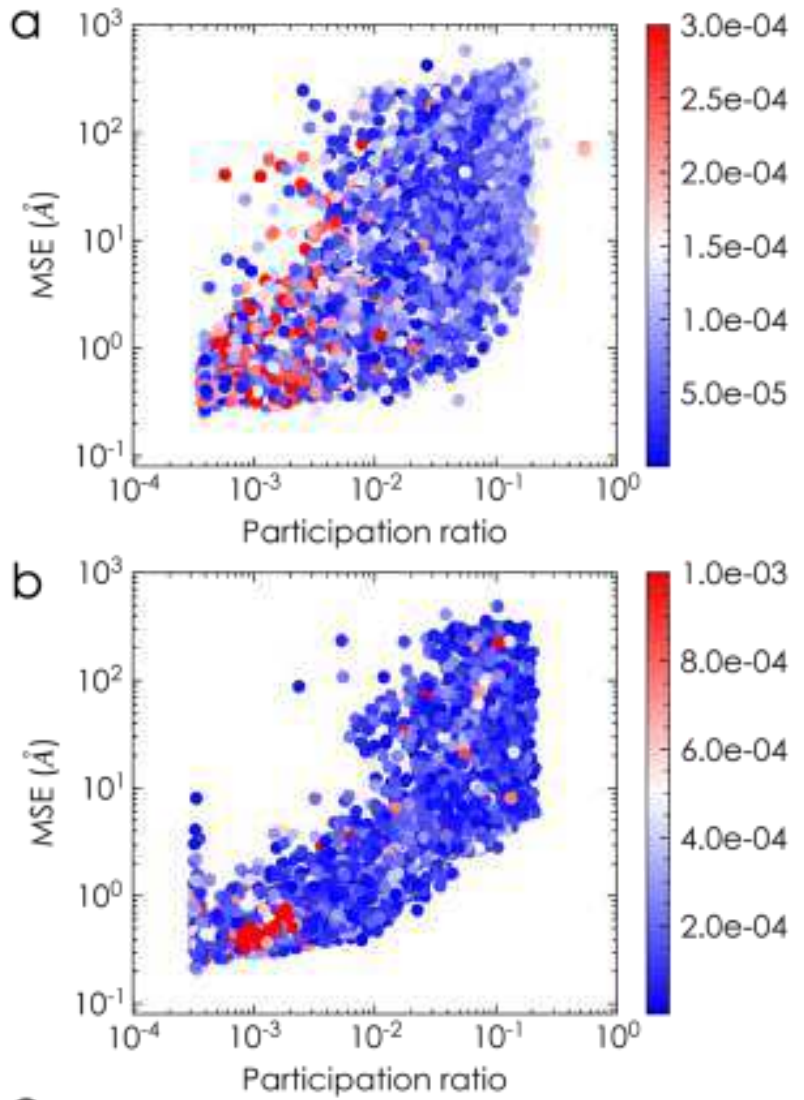

$1.0 \mathrm{e}-03$

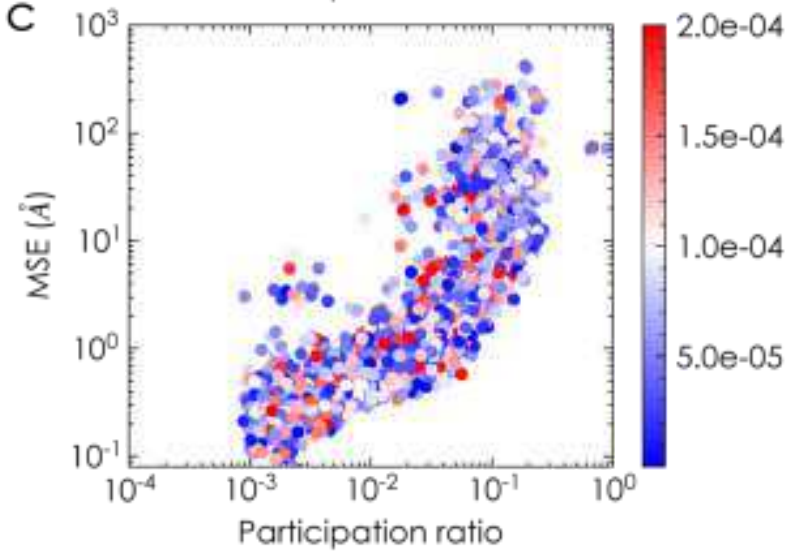

Figure 3| Scattering of modes in coordinates of their PR and MSE. The panel a, b, and c shows the modes in a-PMMA, a-PS, and a-PVC respectively. The grayscale shows the value of modal thermal conductivity in units of $\mathrm{W} \mathrm{m}^{-1} \mathrm{~K}^{-1}$. (Colors online)

11 The large degree of localization observed in amorphous polymers prompted the 12 hypothesis that it may be possible that modes localize onto individual chains where there 
is more homogeneity in bond strength. We therefore evaluated a polymer chain participation ratio (PCPR) as a measure of the involvement of each individual chain in each mode. The definition of PCPR can be found in SI, as it contains a selective summation for PR, that is limited to the atoms on a given polymer chain. Thus, for each polymer chain in a simulation domain, there will be a PCPR value measuring its involvement in a given normal mode. Consequently, we assign M different PCPR values, $\mathrm{PCPR}_{1}, \mathrm{PCPR}_{2}, \ldots$ PCPRM, for each individual mode, where $M$ is the number of polymer chains in the supercell. The subscripts, however, do not denote which specific chain a mode is localized onto. Instead, since all of the chains are the same i.e., chemically indistinguishable, the values of the subscripts are sorted by decreasing value. In this way, the PCPR 1 value for a given mode will always correspond to the chain that has the largest PCPR for that given mode. The PCPR 2 is therefore by definition always smaller than the $\mathrm{PCPR}_{1}$, and it represents the extent of participation the mode has on whichever chain has the $2^{\text {nd }}$ highest participation. The PCPR values then continue in decreasing order of participation, with PCPRM being be the smallest. The PCPR spectrums of three polymers are provided in the SI (see Fig. S3).

With this definition for PCPR, the ratio $\beta_{2}=\mathrm{PCPR} / \mathrm{PCPR} 1$ becomes an important quantity, because it describes the extent to which a mode is localized onto more than one chain. For example, when $\beta_{2}$ is less than 0.1 , it indicates that at least $53 \%$ of the energy of the mode is confined to one polymer chain. Here, it should be noted that there is a somewhat complex relationship between the value of $\beta_{2}$ and the amount of energy confined to a single chain, as explained in the SI. However, most modes with $\beta_{2}<0.1$ have more than $90 \%$ of their energy localized onto a single chain. Similarly, the ratio $\beta_{k}$ will tell whether there are $\mathrm{k}$ chains involved in this mode. The $\beta_{2}$ spectrums of three polymers are shown in Fig. S3d in SI, which suggests a large number of modes have a small $\beta_{2}$. To better understand this, we show the cumulative DOS with respect to $\beta_{2}$ in Fig. $2 \mathrm{~d}$. If we take $\beta_{2}<0.1$, to mean a mode is primarily localized onto a single chain, then for a-PMMA, $52 \%$ of the localized modes are localized onto a single chain, and this fraction is $75 \%$ for a-PS and $74 \%$ for a-PVC. This is a somewhat surprising result, as it shows a strong tendency for modes to localize onto individual chains, which is a special feature of polymers. The strength of covalent bonds are usually three orders of magnitude higher than that of van der Waals interactions. The disparity in bond strength for inter vs. intra chain interactions is rather unique to polymers and possibly some 2D materials (e.g., graphite, $\mathrm{MoS}_{2}, \mathrm{~W}_{2} \mathrm{Se}$, Hexagonal BN etc.). Beside, individual chains have repeating units. Such repeating units together with strong bonds likely give rise to strong localization onto an individual chain. It is therefore a reasonable hypothesis that heat flux will primarily circulate within those homogenous substructures, e.g. polymer chains, and barely transmitted among those substructures.

Conceptually, the homogeneous bond strength and repeating units within an individual chain creates entries within the dynamical matrix of similar magnitude, and presumably the chain/string of atoms that are interconnected with covalent bonds, leads to a natural tendency to delocalize over those bonds for some modes. Conversely, when one encounters the ends of the polymer chains, or monomer's lateral boundaries, there is a discontinuity in bond strength as the next nearest neighboring atoms experience van der 
Waals or electrostatic interactions, which are much weaker. It then stands to reason that this large change in the bond strength tends to terminate the delocalization of modes, as it becomes difficult for non-covalently bonded, yet neighboring atoms, to vibrate together/collectively at the same high frequencies as those associated with the covalently bonded atoms on a different chain. This observation provides intuition on how heat conduction through amorphous polymers occurs. One might expect that these two groups of modes, i.e., modes confined to a single chain, versus modes that are able to spread across multiple chains, might exhibit different transport characteristics, as the latter category are more likely to couple/share energy between among different chains. This point will become clearer when we discuss thermal conductivity.

We emphasize again that a-PMMA, a-PS, and a-PVC have significantly different structures i.e., the monomers are comprised of 15,16, and 6 atoms respectively. PS is the only polymer with an aromatic ring. And the polymers contain different functional groups. However, the observations about the high degree of localization in these polymers are consistent amongst all three and are independent of supercell size (see SI - Fig. S1). Thus, we postulate that it may be also the case that most of the modes in many other amorphous polymers are locons. However, it is not clear to what extent these modes contribute to TC, and if they do, which classifications of modes contribute most (i.e., modes localized onto individual chains or modes delocalized over multiple chains)? Conventional wisdom, based on the PGM, would suggest that localized modes in general do not contribute much to thermal transport, but if they're the predominant mode type in amorphous polymers, it stands to reason that they may be the dominant contributors to TC, simply because they're the dominant mode type.

\section{Contributions to Thermal conductivity}

In Fig. 4 we show the normalized TC accumulation with respect to PR, MSE, and $\beta_{2}$, based on GKMA. Fig.4a shows the locons, defined by $P R<0.1$, contribute $82 \%, 95 \%$, and $98 \%$ to TC for a-PMMA, a-PS, and a-PVC respectively. Therefore, they are the dominant contributors to thermal transport. This is very different than prior studies, some of which have suggested that locons do not contribute significantly, ${ }^{19,21,30,35,46-50}$ and the highest locon contributions reported by Lv \& Henry, ${ }^{35}$ was only $10 \%$. Note that even though locons dominate the population and hence the TC in amorphous polymers, their contributions are small, yet still comparable, on a per mode basis, to the delocalized modes. For example, in a-PMMA delocalized modes contribute 2 times more on a per mode basis, but in a-PS and a-PVC, the delocalized modes only contribute $20 \%$ and $14 \%$ more. This provides significant insight into why amorphous polymer TC is much lower than that of amorphous inorganics, namely because amorphous polymers are predominated by locons, which in general, are poor heat conductors. Similar observations can be made using the measure of MSE as shown in Fig. 4b. Modes that are spatially localized (MSE < $10 \AA$ ) contribute the majority of the TC in amorphous polymers, specifically, $61 \%, 87 \%$, and $88 \%$ for a-PMMA, a-PS, and a-PVC, respectively. This is simply because modes with MSE $<10 \AA$ are the dominate type of modes in amorphous polymers. On a per mode basis, their contributions are smaller yet comparable to the spatially delocalized modes. Figure $4 c$ plots thermal conductivity as a function of $\beta_{2}$ that 
1 represents localized phonons spreading over two chains, and it clearly shows heat is 2 mainly conducted by localized modes able to across chains.
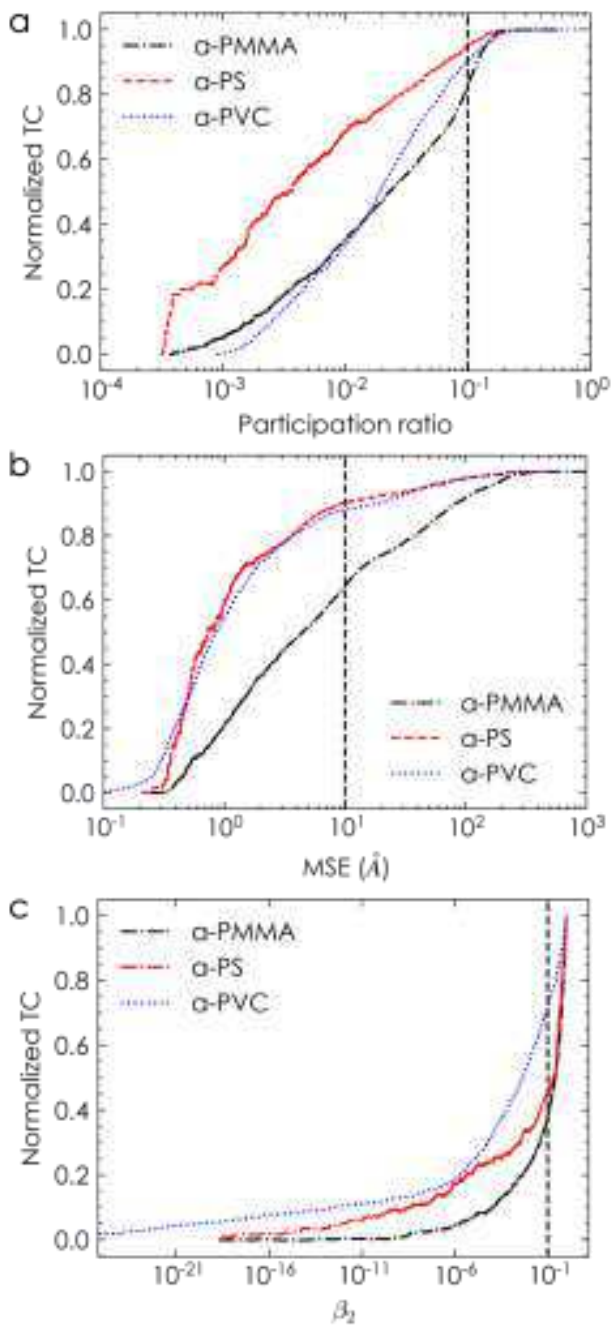

Figure 4| Normalized cumulative TC with respect to modal frequency (panel a), PR (panel $\mathrm{b}$ ), and $\beta_{2}$ (panel $\mathrm{c}$ ). The vertical lines in panel a and b show the separations of locons, while the vertical line in panel c shows the separation of modes confined onto a single chain. For locons defined as modes with PR below 0.1 , they contribute $82 \%, 95 \%$, and $98 \%$ of the total TC in a-PMMA, a-PS, and a-PVC, respectively. (Colors online)

With this mode-resolved TC, we calculated the temperature dependent TC with quantum correction. In Fig. 5, the quantum corrected temperature dependent TC for the three polymers studied is compared with experimental values for a-PMMA, ${ }^{85-87}$ a-PS, ${ }^{88-90}$ and a-PVC. ${ }^{91}$ 


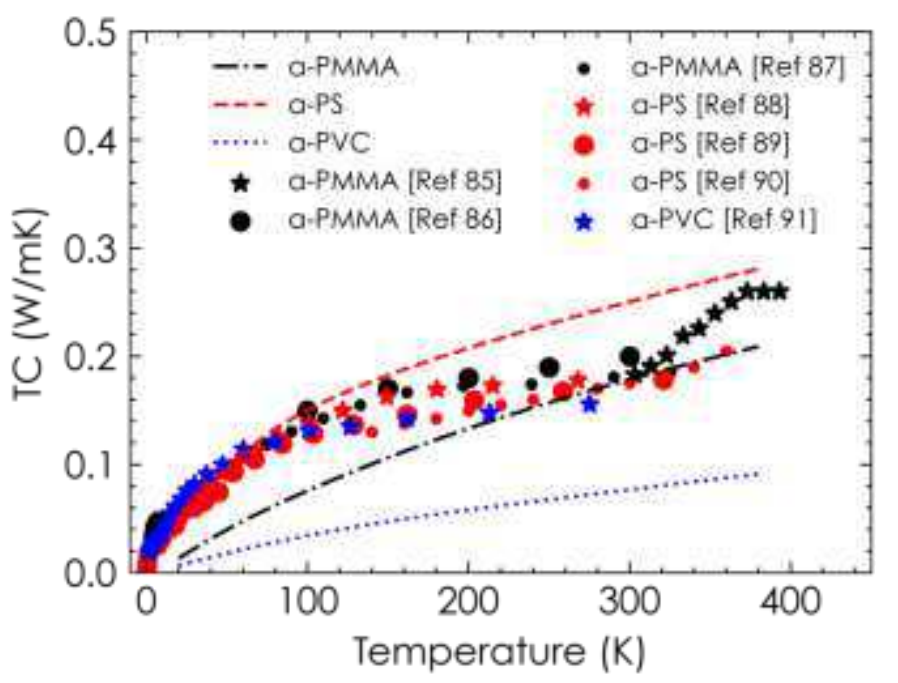

Figure 5| Temperature dependent TC from GKMA with quantum correction. Solid lines are calculated results, dots are experimental values reported in literatures. (Colors online)

The GKMA results show the correct qualitative trends, although there are significant quantitative discrepancies at different temperatures. The calculations underestimate the TC for a-PMMA and a-PVC, while slightly overestimate that of a-PS. We believe the reason can be attributed to a combination of the difference of polymer molecular weights (MW) and size effects. It is known that for polymers, the TC has a strong positive dependence on its MW. ${ }^{92}$ Due to the limitations of computational power, the MW of aPMMA, a-PVC, and a-PS are 5,000, 1,696, and 7,813 $\mathrm{g} / \mathrm{mol}$ in our simulations (every chain contains 200 repeating units), which are orders of magnitudes smaller than the MW of the common products of these polymers. Nevertheless, obtaining good quantitative agreement in temperature dependence of TC is not the main objective here, but the TC results seem to suggest that the mode level observations made in our simulation could still be meaningful and provide important insights for understanding the thermal transport in amorphous polymers.

Although delocalized modes contribute more to TC than locons on a per mode basis, interestingly, the most active modes with the highest TC contributions are strongly localized. As shown in Fig. 3, the modes with highest modal TC are more likely to be localized. It is clear that for all three polymers in study, the modes with highest modal TC appear in the regime with low PR and low MSE. Similar observations are found in the modal TC spectrums shown in SI Fig. S4, where the TC outliers are strongly localized. We summarized the average modal TC value of strongly localized modes (SLM) defined as having $P R<0.01$ and MSE $<3 \AA$, versus that of other modes (non-SLM) that do not satisfy this criterion, in Table1. For all three polymers in study, the average modal TC values of SLM are found to outperform that of non-SLM. 
Table 1| The number and average modal TC values of SLM with $P R<0.01$ and MSE $<3$ $\AA$ and non-SLM modes in amorphous polymers.

\begin{tabular}{|c|c|c|c|}
\hline & a-PMMA & a-PS & a-PVC \\
\hline Fraction of SLM & $42.1 \%$ & $63.5 \%$ & $36.0 \%$ \\
\hline Avg. TC of SLM & $13.2 \times 10^{-5} \mathrm{Wm}^{-1} \mathrm{~K}^{-1}$ & $23.6 \times 10^{-5} \mathrm{Wm}^{-1} \mathrm{~K}^{-1}$ & $8.0 \times 10^{-5} \mathrm{Wm}^{-1} \mathrm{~K}^{-1}$ \\
\hline Fraction of non-SLM & $57.9 \%$ & $36.5 \%$ & $63.0 \%$ \\
\hline Avg. TC of non-SLM & $7.4 \times 10^{-5} \mathrm{Wm}^{-1} \mathrm{~K}^{-1}$ & $16.4 \times 10^{-5} \mathrm{Wm}^{-1} \mathrm{~K}^{-1}$ & $7.8 \times 10^{-5} \mathrm{Wm}^{-1} \mathrm{~K}^{-1}$ \\
\hline SLM/non-SLM & $176 \%$ & $143.9 \%$ & $107.0 \%$ \\
Avg. TC ratio & & & \\
\hline
\end{tabular}

3

4

This finding suggests that there is a group of locons participating in thermal transport more actively than the others. Note that this observation is not in conflict with our previous statement that delocalized modes contribute more to thermal transport on a per mode basis. To explain what is special about these active locons, we studied how the locons are localized in polymer structures. In a-PMMA for example, we notice there are a few modes involving two chains with large $\left(>10^{-5}\right)$ PCPR2's and low $\left(<10^{-10}\right)$ PCPR3's around $50 \mathrm{THz}$, where most modes in the same frequency range are confined to a single chain, and no modes are spread across three or more chains. There are a total of 62 out of 9024 of such modes that are delocalized onto a second chain but not a third chain. Interestingly, the average magnitude of these 62 modes' TC is $18.4 \times 10^{-5} \mathrm{Wm}^{-1} \mathrm{~K}^{-1}$. This value is $60 \%$ higher than the average magnitude of TC for SLM with both a low PR and MSE and nearly twice the average magnitude of all mode in a-PMMA. Generally, we have found most of the locons are confined onto a single chain, as suggested by the more-than-half cumulative contribution to DOS by modes with $\beta_{2}<0.1$ in Fig. $2 \mathrm{~d}$. As shown in the cumulative TC contribution with respect to $\beta_{2}$ in Fig. 4c, however, modes confined onto a single chain only comprise less than half of the total TC, despite their populations being more than half (Fig. 2d). Even if we exclude the contributions from the minority of delocalized modes and focus on the locons, the contribution to TC from locons localized onto multiple chains (i.e., for modes with $\mathrm{PR}<0.1$ yet $\beta_{2}>0.1$ ), is still higher than the modes localized onto a single chain, as summarized in Tab. 2. The unbalanced contribution in DOS and TC suggests that the locons localized onto multiple chains contribute more than the Icoons localized onto a single chain.

Table 2| The cumulative DOS and TC contribution of locons localized onto multiple chains.

\begin{tabular}{|c|c|c|c|}
\hline & a-PMMA & a-PS & a-PVC \\
\hline Cumulative DOS & $48.4 \%$ & $24.7 \%$ & $28.6 \%$ \\
\hline Cumulative TC & $54.2 \%$ & $52.9 \%$ & $29.2 \%$ \\
\hline
\end{tabular}

Therefore, we conclude that the modes with very large TC values are more likely to be strongly localized modes and as a result, the average TC value of the strongly localized modes (SLM) is slightly higher than other modes. And the locons that spread onto multiple chains contribute more to thermal transport than the ones confined onto single chains, 
even though the later type of locons are the majority in amorphous polymers. These findings differ from the behavior of locons in other disordered materials.

\section{Conclusions}

In this work, we studied the normal modes and TC of three amorphous polymers (i.e., aPMMA, a-PS, and a-PVC) using LD and GKMA. The results show that in all three amorphous polymers studied, locons are the predominant mode type, comprising more than $90 \%$ of the modes. GKMA results showed that even though locons in previously studied systems have exhibited negligible/small contributions, here, since they are the predominant mode type, their contributions comprise more than $80 \%$ of the TC. This shows that locons can play an important role in amorphous polymers, although their contributions are much smaller on a per mode basis, by comparison to delocalized modes. Deeper investigation of the character of locon modes showed that $10-30 \%$ still involve some participation of atoms over a large region. Such modes are still locons in the sense that their energy is concentrated on a small subset of atoms, but this energy can still be spread over a spatial region comparable to the size of the supercell (i.e., > 10A). The introduction of a new descriptor, termed the MSE facilitated quantification of the length scale over which such modes are spread.

Further modal analysis showed that a major fraction of the localized modes are localized onto a single polymer chain, as quantified by the value $\beta_{2}$. This is presumed to be caused by the inhomogeneity in bond strength between intra and inter chain interactions. Calculations of $\beta_{2}$ for the different polymers showed that for more than half of the localized modes, greater than half of their energy was confined to a single polymer chain. The minority of locons that are nested onto multiple chains, however, have a larger average TC than the main type of locons that are localized onto a single chain, suggesting that these modes serve as bridges across the chain-chain boundaries through van der Waals interaction. Interestingly, this seems to be an important heat transport channel for amorphous polymers, even though it happens through locons.

Furthermore, the localized nature of the modes in amorphous polymers offers an explanation for why their TC is about an order of magnitude smaller than that of amorphous semiconductors. Notably, since localized mode contributions are in general smaller than that of delocalized modes, having a material with predominantly locons translates to an expectation of low TC. Thus, the results herein have provided several new insights in amorphous polymers, namely: (1) that most of the modes are locons, of which a large fraction are localized onto individual chains; (2) Strongly localized modes can still be active in thermal transport by having the highest modal TC values in amorphous polymers; (3) Although most modes are localized, the modes with the largest contributions to TC are modes that span more than one chain and conceivably serve as bridges between chains, implying that the bottleneck for transport is the weaker interchain interactions; (4) Amorphous polymer TC is likely lower than that of amorphous semiconductors, in general, because of the large number of locons. It then follows as a natural hypothesis that what likely causes the dramatic increase in TC that can occur with mechanical stretching of polymers, is the conversion of locons to diffusons and propagons, 
which correspondingly have larger contributions on a per mode basis. It is from this perspective, that considerable follow-up work is needed to better understand the extent to which the aforementioned findings are generalizable to amorphous thermoplastics, and even possibly some thermosets.

\section{Acknowledgements}

A.H. and F.D. acknowledge support from the National Science Foundation (NSF) career award to A.H. (award no. 1554050), the Office of Naval Research (ONR) under a MURI program (grant no. N00014-18-1-2429). B.L. and G.C. acknowledge support from the U.S. Department of Energy (DOE)-Basic Energy Sciences (Award No. DE-FG0202ER45977).

\section{Contributions}

F.D., B.L., and A.H. conceived the idea and supervised the work, along with G.C. F.D. and B.L. performed the simulations. All authors contributed to the analyses, discussions, and writing of the manuscript.

\section{References}

1. Wei, X., Wang, Z., Tian, Z. \& Luo, T. Thermal Transport in Polymers: A Review. J. Heat Transfer 143, 1ENG (2021).

2. Henry, A. THERMAL TRANSPORT IN POLYMERS. Annu. Rev. Heat Transf. 17, 485-520 (2014).

3. Qian, X., Zhou, J. \& Chen, G. Phonon-engineered extreme thermal conductivity materials. Nat. Mater. 2021209 20, 1188-1202 (2021).

4. Choy, C. L. Thermal conductivity of polymers. Polymer (Guildf). 18, 984-1004 (1977).

5. Choy, C. L., Chen, F. C. \& Luk, W. H. Thermal conductivity of oriented crystalline polymers. J. Polym. Sci. Polym. Phys. Ed. 18, 1187-1207 (1980).

6. Shen, S., Henry, A., Tong, J., Zheng, R. \& Chen, G. Polyethylene nanofibres with very high thermal conductivities. Nat. Nanotechnol. 5, 251-255 (2010).

7. $\mathrm{Xu}, \mathrm{Y}$. et al. Molecular engineered conjugated polymer with high thermal conductivity. Sci. Adv. 4, eaar3031 (2018).

8. Shulumba, N., Hellman, O. \& Minnich, A. J. Lattice Thermal Conductivity of Polyethylene Molecular Crystals from First-Principles Including Nuclear Quantum Effects. Phys. Rev. Lett. 119, 185901 (2017).

9. Wang, X., Kaviany, M. \& Huang, B. Phonon coupling and transport in individual polyethylene chains: A comparison study with the bulk crystal. Nanoscale $\mathbf{9}$, 18022-18031 (2017).

10. Shrestha, R. et al. Crystalline polymer nanofibers with ultra-high strength and thermal conductivity. Nat. Commun. 9, 1-9 (2018).

11. Henry, A. \& Chen, G. High thermal conductivity of single polyethylene chains using molecular dynamics simulations. Phys. Rev. Lett. 101, 235502 (2008).

12. Cevallos, J. G., Bergles, A. E., Bar-Cohen, A., Rodgers, P. \& Gupta, S. K. 
Polymer heat exchangers-history, opportunities, and challenges. Heat Transfer Engineering vol. 33 1075-1093 (2012).

13. Clemens, W. Polymer electronics. in Technology Guide: Principles - Applications Trends 84-87 (Springer Berlin Heidelberg, 2009). doi:10.1007/978-3-540-88546717.

14. Wan, Y. J. et al. Recent advances in polymer-based electronic packaging materials. Composites Communications vol. 19 154-167 (2020).

15. PEIERLS, R. On the Kinetic Theory of Thermal Conduction in Crystals. in 15-48 (1997). doi:10.1142/9789812795779_0004.

16. Chen, G. P. Nanoscale energy transport and conversion : a parallel treatment of electrons, molecules, phonons, and photons. (Oxford University Press, 2005).

17. Zhang, Z. M. Nano/Microscale Heat Transfer. (Springer International Publishing, 2020). doi:10.1007/978-3-030-45039-7.

18. Klemens, P. G. The scattering of low-frequency lattice waves by static imperfections. Proc. Phys. Soc. Sect. A 68, 1113-1128 (1955).

19. Allen, P. B. \& Feldman, J. L. Thermal conductivity of disordered harmonic solids. Phys. Rev. B 48, 12581-12588 (1993).

20. Sun, T. \& Allen, P. B. Lattice thermal conductivity: Computations and theory of the high-temperature breakdown of the phonon-gas model. Phys. Rev. B - Condens. Matter Mater. Phys. 82, 224305 (2010).

21. Lv, W. \& Henry, A. Examining the Validity of the Phonon Gas Model in Amorphous Materials. Sci. Rep. 6, 1-8 (2016).

22. Seyf, H. R. et al. Rethinking phonons: The issue of disorder. npj Comput. Mater. 3, 49 (2017).

23. The Physics of Phonons - 1st Edition - G.P Srivastava - Routledge Boo. https://www.routledge.com/The-Physics-ofPhonons/Srivastava/p/book/9780852741535.

24. Garg, J., Bonini, N., Kozinsky, B. \& Marzari, N. Role of disorder and anharmonicity in the thermal conductivity of silicon-germanium alloys: A firstprinciples study. Phys. Rev. Lett. 106, 045901 (2011).

25. Tian, Z., Lee, S. \& Chen, G. Heat transfer in thermoelectric materials and devices. J. Heat Transfer 135, 103 (2013).

26. Esfarjani, K., Garg, J. \& Chen, G. MODELING HEAT CONDUCTION FROM FIRST PRINCIPLES. Annu. Rev. Heat Transf. 17, 9-47 (2014).

27. Feng, T. \& Ruan, X. Prediction of spectral phonon mean free path and thermal conductivity with applications to thermoelectrics and thermal management: A review. Journal of Nanomaterials vol. 2014 (2014).

28. Lindsay, L., Broido, D. A. \& Reinecke, T. L. Ab initio thermal transport in compound semiconductors. Phys. Rev. B - Condens. Matter Mater. Phys. 87, 165201 (2013).

29. Tadano, T., Gohda, Y. \& Tsuneyuki, S. Anharmonic force constants extracted from first-principles molecular dynamics: Applications to heat transfer simulations. J. Phys. Condens. Matter 26, 225402 (2014).

30. Allen, P. B., Feldman, J. L., Fabian, J. \& Wooten, F. Diffusons, Locons, Propagons: Character of Atomic Vibrations in Amorphous Si. Philos. Mag. B 79, 1715-1731 (1999). 
31. Wang, X. \& Huang, B. Computational study of in-plane phonon transport in Si thin films. Sci. Rep. 4, 1-10 (2014).

32. Shiomi, J., Esfarjani, K. \& Chen, G. Thermal conductivity of half-Heusler compounds from first-principles calculations. Phys. Rev. B - Condens. Matter Mater. Phys. 84, 104302 (2011).

33. McGaughey, A. J. H. \& Kaviany, M. Phonon transport in molecular dynamics simulations: Formulation and thermal conductivity prediction. Adv. Heat Transf. 39, 169-255 (2006).

34. Baldi, G. et al. Thermal conductivity and terahertz vibrational dynamics of vitreous silica. Phys. Rev. B - Condens. Matter Mater. Phys. 77, 214309 (2008).

35. Lv, W. \& Henry, A. Non-negligible Contributions to Thermal Conductivity from Localized Modes in Amorphous Silicon Dioxide. Sci. Rep. 6, 1-8 (2016).

36. Agne, M. T., Hanus, R. \& Snyder, G. J. Minimum thermal conductivity in the context of: Diffuson -mediated thermal transport. Energy Environ. Sci. 11, 609616 (2018).

37. Jugdersuren, B. et al. Thermal conductivity of amorphous and nanocrystalline silicon films prepared by hot-wire chemical-vapor deposition. Phys. Rev. B 96, 014206 (2017).

38. Moon, J., Latour, B. \& Minnich, A. J. Propagating elastic vibrations dominate thermal conduction in amorphous silicon. Phys. Rev. B 97, 024201 (2018).

39. Allen, P. B., Du, X., Mihaly, L. \& Forro, L. Thermal conductivity of insulating $\mathrm{Bi2Sr2YCu2O} 8$ and superconducting Bi2Sr2CaCu2O8: Failure of the phonon-gas picture. Phys. Rev. B 49, 9073-9079 (1994).

40. Charles Kittel. Introduction to Solid State Physics. (Wiley, 2004).

41. DeAngelis, F. et al. Thermal Transport in Disordered Materials. Nanoscale Microscale Thermophys. Eng. 23, 81-116 (2019).

42. Feldman, J. L., Kluge, M. D., Allen, P. B. \& Wooten, F. Thermal conductivity and localization in glasses: Numerical study of a model of amorphous si\&icon. PHYSICAL REVIEW B vol. 48.

43. Allen, P. B., Feldman, J. L., Fabian, J. \& Wooten, F. Diffusons, locons and propagons: Character of atomie yibrations in amorphous Si. Philos. Mag. B Phys. Condens. Matter; Stat. Mech. Electron. Opt. Magn. Prop. 79, 1715-1731 (1999).

44. Seyf, H. R. \& Henry, A. A method for distinguishing between propagons, diffusions, and locons. J. Appl. Phys. 120, 025101 (2016).

45. Moon, J. \& Minnich, A. J. Sub-amorphous thermal conductivity in amorphous heterogeneous nanocomposites. RSC Adv. 6, 105154-105160 (2016).

46. Ni, H., Li, X. \& Gao, H. Elastic modulus of amorphous $\mathrm{SiO} 2$ nanowires. Appl. Phys. Lett. 88, 1-3 (2006).

47. Alam, M. T., Manoharan, M. P., Haque, M. A., Muratore, C. \& Voevodin, A. Influence of strain on thermal conductivity of silicon nitride thin films. $J$. Micromechanics Microengineering 22, 8 (2012).

48. Alam, A., Chouhan, R. K. \& Mookerjee, A. Thermal conductivity and diffusionmediated localization in Fe 1-xCr x alloys from first principles. Phys. Rev. B Condens. Matter Mater. Phys. 84, 224309 (2011).

49. Yu, X. \& Leitner, D. M. Thermal conductivity computed for vitreous silica and methyl-doped silica above the plateau. Phys. Rev. B - Condens. Matter Mater. 
Phys. 74, 184305 (2006).

50. Leitner, D. M. Vibrational energy transfer and heat conduction in a onedimensional glass. Phys. Rev. B - Condens. Matter Mater. Phys. 64, 094201 (2001).

51. Shenogin, S., Bodapati, A., Keblinski, P. \& McGaughey, A. J. H. Predicting the thermal conductivity of inorganic and polymeric glasses: The role of anharmonicity. J. Appl. Phys. 105, 034906 (2009).

52. Krivchikov, A. I. et al. Effects of internal molecular degrees of freedom on the thermal conductivity of some glasses and disordered crystals. Phys. Rev. B Condens. Matter Mater. Phys. 85, 014206 (2012).

53. Schober, H. R., Buchenau, U. \& Gurevich, V. L. Pressure dependence of the boson peak in glasses: Correlated and uncorrelated perturbations. Phys. Rev. B Condens. Matter Mater. Phys. 89, 014204 (2014).

54. Schön, J. C. Simulation of thermal conductivity and heat transport in solids. Phys. Rev. B - Condens. Matter Mater. Phys. 59, 4125-4133 (1999).

55. Courtens, E., Foret, M., Hehlen, B. \& Vacher, R. Vibrational modes of glasses. Solid State Commun. 117, 187-200 (2001).

56. Parshin, D. A. \& Laermans, C. Interaction of quasilocal harmonic modes and boson peak in glasses. Phys. Rev. B - Condens. Matter Mater. Phys. 63, 132203 (2001).

57. Crupi, C., D’angelo, G., Tripodo, G., Carini, G. \& Bartolotta, A. Low-temperature specific heat in caesium borate glasses. Philos. Mag. 87, 741-747 (2007).

58. Schirmacher, W. Thermal conductivity of glassy materials and the 'boson peak'. Europhys. Lett. 73, 892-898 (2006).

59. Zhou, Z. et al. On the existence of Einstein oscillators and thermal conductivity in bulk metallic glass. Appl. Phys. Lett. 89, 031924 (2006).

60. Schirmacher, W., Ruocco, G. \& Scopigno, T. Acoustic attenuation in glasses and its relation with the boson peak. Phys. Rev. Lett. 98, 025501 (2007).

61. D'Angelo, G. et al. Boson peak in alkaline borate glasses: Raman spectroscopy, neutron scattering, and specific-heat measurements. Phys. Rev. B - Condens. Matter Mater. Phys. 79, 014206 (2009).

62. Kwon, S., Zheng, J., Wingert, M. C., Cui, S. \& Chen, R. Unusually High and Anisotropic Thermal Conductivity in Amorphous Silicon Nanostructures. ACS Nano 11, 2470-2476 (2017).

63. Fabian, J., Feldman, J. L., Hellberg, C. S. \& Nakhmanson, S. M. Numerical study of anharmonic vibrational decay in amorphous and paracrystalline silicon. Phys. Rev. B - Condens. Matter Mater. Phys. 67, 224302 (2003).

64. Liu, X. et al. High thermal conductivity of a hydrogenated amorphous silicon film. Phys. Rev. Lett. 102, 035901 (2009).

65. He, Y., Donadio, D. \& Galli, G. Heat transport in amorphous silicon: Interplay between morphology and disorder. Appl. Phys. Lett. 98, 144101 (2011).

66. Yang, H.-S. et al. Anomalously high thermal conductivity of amorphous $\mathrm{Si}$ deposited by hot-wire chemical vapor deposition. doi:10.1103/PhysRevB.81.104203.

67. He, Y., Savić, I., Donadio, D. \& Galli, G. Lattice thermal conductivity of semiconducting bulk materials: Atomistic simulations. Phys. Chem. Chem. Phys. 
14, 16209-16222 (2012).

68. Zhan, T. et al. Phonons with long mean free paths in a-Si and a-Ge. Appl. Phys. Lett. 104, 071911 (2014).

69. Braun, J. L. et al. Size effects on the thermal conductivity of amorphous silicon thin films. Phys. Rev. B 93, 140201 (2016).

70. Wang, Y., Yang, F. \& Xiao, P. Glass-like thermal conductivities in (La 1-x1 Y x1) 2 $(\operatorname{Zr} 1-\mathrm{x} 2 \mathrm{Y} \times 2) 207-\mathrm{x} 2(\mathrm{x}=\mathrm{x} 1+\mathrm{x} 2,0 \leq \mathrm{x} \leq 1.0)$ solid solutions. Acta Mater. 60, 7024-7033 (2012).

71. Giri, A., Braun, J. L., Rost, C. M. \& Hopkins, P. E. On the minimum limit to thermal conductivity of multi-atom component crystalline solid solutions based on impurity mass scattering. Scr. Mater. 138, 134-138 (2017).

72. Konstantinov, V. A., Manzhelii, V. G., Revyakin, V. P., Sagan, V. V. \& Pursky, O. I. Isochoric thermal conductivity of solid carbon oxide: The role of phonons and 'diffusive' modes. J. Phys. Condens. Matter 18, 9901-9909 (2006).

73. Chiritescu, C. et al. Ultralow thermal conductivity in disordered, layered WSe2 crystals. Science (80-. ). 315, 351-353 (2007).

74. Korolyuk, O. A., Krivchikov, A. I., Sharapova, I. V. \& Romantsova, O. O. Heat transfer in solid methyl alcohol. in Low Temperature Physics vol. 35 290-293 (American Institute of Physics Inc., 2009).

75. Giri, A., Hopkins, P. E., Wessel, J. G. \& Duda, J. C. Kapitza resistance and the thermal conductivity of amorphous superlattices. J. Appl. Phys. 118, 165303 (2015).

76. Tlili, A. et al. Thermal transport properties in amorphous/nanocrystalline metallic composites: A microscopic insight. Acta Mater. 136, 425-435 (2017).

77. Sosso, G. C., Donadio, D., Caravati, S., Behler, J. \& Bernasconi, M. Thermal transport in phase-change materials from atomistic simulations. Phys. Rev. B Condens. Matter Mater. Phys. 86, 104301 (2012).

78. Wang, Y., Song, Z. \& Xu, Z. Mechanistic transition of heat conduction in twodimensional solids: A study of silica bilayers. Phys. Rev. B - Condens. Matter Mater. Phys. 92, 245427 (2015).

79. Zhu, T. \& Ertekin, E. Phonons, Localization, and Thermal Conductivity of Diamond Nanothreads and Amorphous Graphene. Nano Lett. 16, 4763-4772 (2016).

80. Lv, W. \& Henry, A. Direct calculation of modal contributions to thermal conductivity via Green-Kubo modal analysis. New J. Phys. 18, 13028 (2016).

81. Lv, W. \& Henry, A. Phonon transport in amorphous carbon using Green - Kubo modal analysis. Appl. Phys. Lett. 108, 181905 (2016).

82. Seyf, H. R., Gordiz, K., Deangelis, F. \& Henry, A. Using Green-Kubo modal analysis (GKMA) and interface conductance modal analysis (ICMA) to study phonon transport with molecular dynamics. J. Appl. Phys. 125, 081101 (2019).

83. Seyf, H. R., Gordiz, K., Deangelis, F. \& Henry, A. Using Green-Kubo modal analysis (GKMA) and interface conductance modal analysis (ICMA) to study phonon transport with molecular dynamics. J. Appl. Phys. 125, 081101 (2019).

84. Murphy, N. C., Wortis, R. \& Atkinson, W. A. Generalized Inverse Participation Ratio as a Possible Measure of Localization for Interacting Systems. Phys. Rev. B - Condens. Matter Mater. Phys. 83, (2010). 
85. Agarwal, S., Saxena, N. S. \& Kumar, V. Temperature Dependence Thermal Conductivity of ZnS/PMMA Nanocomposite. in 737-739 (Springer, Cham, 2014). doi:10.1007/978-3-319-03002-9_190.

86. Reese, W. Temperature Dependence of the Thermal Conductivity of Amorphous Polymers: Polymethyl Methacrylate. J. Appl. Phys. 37, 3227 (1966).

87. Choy, C. L. Thermal conductivity of polymers. Polymer vol. 18 984-1004 (1977).

88. Kommandur, S. \& Yee, S. K. An empirical model to predict temperaturedependent thermal conductivity of amorphous polymers. J. Polym. Sci. Part B Polym. Phys. 55, 1160-1170 (2017).

89. Freeman, J. J. \& Anderson, A. C. Thermal conductivity of amorphous solids. Phys. Rev. B 34, 5684-5690 (1986).

90. Tillmann, A. R., Borges, V. L., Guimarães, G., De Lima e Silva, A. L. F. \& De Lima e Silva, S. M. M. Identification of temperature-dependent thermal properties of solid materials. J. Brazilian Soc. Mech. Sci. Eng. 30, 269-278 (2008).

91. Choy, C. L. \& Greig, D. The low temperature thermal conductivity of isotropic and oriented polymers. J. Phys. C Solid State Phys. 10, 169-179 (1977).

92. Henry, A. THERMAL TRANSPORT IN POLYMERS. Annu. Rev. Heat Transf. 17, 485-520 (2013). 


\section{Supplementary Files}

This is a list of supplementary files associated with this preprint. Click to download.

- polymerPaperSlcp.docx 